\title{
Understanding Growth Patterns of Signet Ring Cell Carcinoma of the Stomach Is Necessary for Successful Endoscopic Resection
}

\author{
Gwang Ha Kim \\ Department of Internal Medicine, Pusan National University School of Medicine and Biomedical Research Institute, Pusan National University \\ Hospital, Busan, Korea
}

See "Growth Patterns of Signet Ring Cell Carcinoma of the Stomach for Endoscopic Resection" by Hyunki Kim, et al. on page 720-726, Vol. 9. No. 6, 2015

Endoscopic resection (ER) has been widely accepted as a minimally invasive treatment for early gastric cancer (EGC) that has a negligible risk of lymph node metastasis. In the past, ER was performed mainly in differentiated-type EGCs, but recently, the indications for ER have been extended to include undifferentiated-type EGCs. ${ }^{1}$ According to the Japanese Gastric Cancer Association, undifferentiated-type gastric carcinoma includes poorly differentiated adenocarcinoma, signet ring cell carcinoma (SRCC), and mucinous adenocarcinoma as defined by the World Health Organization classification. ${ }^{2}$ Several recent studies have shown that patients with undifferentiated-type EGC can be candidates for endoscopic submucosal dissection under specific conditions where there is an acceptably low rate of lymph node metastasis, compatible with that of differentiated-type EGC. ${ }^{3-5}$

In general, ER has been performed for SRCC considered to be a type of undifferentiated adenocarcinoma. However, there are distinct differences in the clinicopathological features according to the subtype of undifferentiated adenocarcinoma. In particular, one of the important features for predicting successful ER is that poorly differentiated adenocarcinoma tends to invade vertically and SRCC tends to spread horizontally. This can lead to different patterns of ER outcomes between poorly differentiated adenocarcinoma and SRCC because histologically incomplete resections in poorly differentiated adenocarcinoma are mainly due to vertical involvement, whereas those in SRCC are mainly due to horizontal involvement of tumor cells. ${ }^{6}$ This growth pattern indicates that the difficulty in endoscopic prediction of the tumor margins in SRCC is due to the subepithelial spreading tendency of SRCC beneath intact the surface epithelium, ${ }^{7}$ which results in underestimation of the true histopathological margins of SRCC.

In an effort to study this phenomenon, Kim et al. ${ }^{8}$ investigated the intramucosal spreading patterns of SRCC in surgical and ER specimens. In this study, the intramucosal spreading patterns were classified into two types: expanding and infiltrative types. The expanding type was defined as a tumor that had a margin that was clearly lined with nonneoplastic mucosa (an epithelial spreading pattern), and the infiltrative type was defined as a tumor that showed diffusely spreading tumor cells (a subepithelial spreading pattern). Thus, the surrounding mucosal pattern differed between the two types. The surrounding mucosa in the infiltrated type was more commonly associated with atrophy, intestinal metaplasia, lack of neutrophil infiltration, and an absence of Helicobacter pylori. Therefore, the authors suggested the importance of the surrounding mucosa as a mechanical barrier for tumor cell spread in SRCC. In a weak barrier state, such as in atrophy or lack of neutrophil infiltration, the tumor cells might tend to have a subepithelial growth pattern. In addition, they showed additional data in ER specimens supporting the above-mentioned assumption, namely, that the positive rate of horizontal margins in ER specimens was higher in the infiltrative type than in the expanding type.

If so, how can we exactly delineate the horizontal margins of SRCC using endoscopy? This process is very important for successful ER of SRCC. Most expert endoscopists agree that it is very difficult, sometimes impossible, to delineate the exact hori-

Correspondence to: Gwang Ha Kim

Department of Internal Medicine, Pusan National University School of Medicine and Biomedical Research Institute, Pusan National University Hospital, 179 Gudeok-ro, Seo-gu, Busan 49241, Korea

Tel: +82-51-240-7869, Fax: +82-51-244-8180, E-mail: doc0224@pusan.ac.kr pISSN 1976-2283 eISSN 2005-1212 http://dx.doi.org/10.5009/gnl15151

@) This is an Open Access article distributed under the terms of the Creative Commons Attribution Non-Commercial License (http://creativecommons.org/licenses/by-nc/4.0) which permits unrestricted non-commercial use, distribution, and reproduction in any medium, provided the original work is properly cited. 
zontal margins of SRCC using endoscopy alone. Accordingly, endoscopic biopsies taken outside the lesion (usually four-quadrant, apart from the endoscopically predicted horizontal margins) are strongly recommended for the accurate determination of the tumor margin before ER. ${ }^{3}$ Several studies have shown the efficacy of acetic acid-indigo carmine chromoendoscopy and magnifying endoscopy with narrow-band imaging to identify the horizontal margins of differentiated-type carcinoma. ${ }^{9-11}$ However, these techniques do not give additional information to delineate the horizontal margins of undifferentiated-type carcinoma, especially SRCC, because of the subepithelial spreading tendency of SRCC.,11

Considering these circumstances, the findings of Kim et al. that the status of the surrounding mucosa can be predictive of the intramucosal spreading patterns in SRCC could give more information to an endoscopist trying to achieve successful ER for SRCC. However, this study has some limitations; the number of included cases was relatively small, and almost all cases included in this study were pure SRCC, not combined with other histologies. Therefore, further large-scale multicenter studies, including studies with a prospective design, are necessary before clinical application of this information.

\section{CONFLICTS OF INTEREST}

No potential conflict of interest relevant to this article was reported.

\section{REFERENCES}

1. Hirasawa T, Gotoda T, Miyata S, et al. Incidence of lymph node metastasis and the feasibility of endoscopic resection for undifferentiated-type early gastric cancer. Gastric Cancer 2009;12:148-152.
2. Japanese Gastric Cancer Association. Japanese Classification of Gastric Carcinoma: 2nd English edition. Gastric Cancer 1998;1:1024.

3. Abe S, Oda I, Suzuki H, et al. Short- and long-term outcomes of endoscopic submucosal dissection for undifferentiated early gastric cancer. Endoscopy 2013;45:703-707.

4. Okada K, Fujisaki J, Yoshida T, et al. Long-term outcomes of endoscopic submucosal dissection for undifferentiated-type early gastric cancer. Endoscopy 2012;44:122-127.

5. Kim DY, Hong SJ, Cho GS, et al. Long-term efficacy of endoscopic submucosal dissection compared with surgery for early gastric cancer: a retrospective cohort study. Gut Liver 2014;8:519-525.

6. Kim JH, Lee YC, Kim H, et al. Endoscopic resection for undifferentiated early gastric cancer. Gastrointest Endosc 2009;69:e1-e9.

7. Kumarasinghe MP, Lim TK, Ooi CJ, Luman W, Tan SY, Koh M. Tubule neck dysplasia: precursor lesion of signet ring cell carcinoma and the immunohistochemical profile. Pathology 2006;38:468471

8. Kim H, Kim JH, Lee YC, et al. Growth patterns of signet ring cell carcinoma of the stomach for endoscopic resection. Gut Liver 2015;9:720-726.

9. Lee BE, Kim GH, Park DY, et al. Acetic acid-indigo carmine chromoendoscopy for delineating early gastric cancers: its usefulness according to histological type. BMC Gastroenterol 2010;10:97.

10. Kiyotoki S, Nishikawa J, Satake M, et al. Usefulness of magnifying endoscopy with narrow-band imaging for determining gastric tumor margin. J Gastroenterol Hepatol 2010;25:1636-1641.

11. Nagahama T, Yao K, Maki S, et al. Usefulness of magnifying endoscopy with narrow-band imaging for determining the horizontal extent of early gastric cancer when there is an unclear margin by chromoendoscopy (with video). Gastrointest Endosc 2011;74:1259-1267. 